\title{
Research on sound absorption performance and optimal design of acoustic materials containing cavity structure
}

\author{
Hao Song ${ }^{1, *}$, Zixian $\mathrm{Cui}^{1}$, Jun $\mathrm{Li}^{1}$, and Buchao $\mathrm{An}^{1}$ \\ ${ }^{1}$ Systems Engineering Research Institute, Fengxian east road No.1, Haidian district, Beijing, China
}

\begin{abstract}
Based on the finite element method, this paper first discusses the sound absorption characteristics of acoustic materials with a cavity structure backed by air, and then takes the internal cavity of the acoustic material and the acoustic material as the research object, considering the sound waves of different frequencies and different incident angles. The maximum sound absorption coefficient is used as the objective function to optimize the design of the cavity acoustic material. The calculation results show that the sound absorption performance of the optimized acoustic material has been significantly improved.
\end{abstract}

\section{Introduction}

Laying sound-absorbing acoustic materials on the surface of underwater structures is a common method to improve its acoustic stealth performance [1]. Rubber, as a material with similar impedance to water, is widely used to make sound-absorbing acoustic materials [2]. However, for a single uniform material, the design of acoustic materials is limited by the contradiction between material parameters and impedance matching. In order to enhance the sound absorption performance of acoustic materials, periodic cavities are usually opened in the acoustic material, through cavity resonance and wave type conversion. , Acoustic scattering between cavities, etc., increase the loss of acoustic energy [3-4]. Through the efforts of scholars from various countries, analytical methods have been used to calculate the acoustic characteristics of sound-absorbing acoustic materials, and rich results have been achieved. Based on the transfer function method, Sharma GS et al. equated the cavity acoustic material to a uniform material and calculated its sound absorption coefficient [5-7]. Changzheng Ye et al. [8] studied the acoustic performance of sound-absorbing acoustic materials with variable cross-section cylindrical cavities, and compared the sound-absorbing performance of acoustic materials with different cavity types. Ivansson SM et al. [9] used multiple scattering methods to solve the sound absorption characteristics of cavity acoustic materials. However, the analytical method is only effective for some orthogonal cavity forms.

The finite element method is not limited by the geometric configuration of the structure. It is commonly used to calculate the acoustic properties of sound-absorbing acoustic

*Corresponding author: songhaox@163.com 
materials. Hennion [10] uses the finite element method to study the reflection and transmission characteristics of periodic cylindrical cavity acoustic materials, Tan Hongbo [11] The sound absorption properties of acoustic materials containing spherical, cylindrical and conical cavities are studied. With the development of underwater acoustic technology, the requirements for the acoustic performance of acoustic materials have become higher and higher, and the existing cavity types can no longer meet the requirements for use. Therefore, domestic researchers began to study the optimization design of acoustic materials, Ivansson SM [12] Did the optimization of acoustic materials with fixed cavity shapes. Shang Chao [13] optimized the design of the hybrid cavity structure.

Although the above optimization research has improved the sound absorption capacity of acoustic materials to a certain extent, the optimization object is relatively single, and the optimization result does not represent the optimal result. This article focuses on acoustic materials with internal cavities, with the largest sound absorption coefficient As the objective function, considering the incident waves of different frequencies and different angles, the Nelder-Mead algorithm is used to optimize the design of the acoustic material cavity line and the acoustic material material.

\section{Finite element and optimization algorithm theory}

\subsection{Sound field fluid-structure coupling}

In this paper, the finite element method is used to calculate the sound absorption performance of the acoustic material with cavity, considering the fluid-solid coupling problem, the elastic body and fluid coupling finite element form, there are:

$$
\left(\begin{array}{cc}
{\left[K_{T}\right]-\omega^{2}\left[M_{T}\right]} & -\left[L_{\mathrm{c}}\right]^{T} \\
-\rho_{S} \omega^{2}\left[L_{\mathrm{c}}\right] & {\left[K_{S}\right]-\omega^{2}\left[M_{S}\right]}
\end{array}\right)\left(\begin{array}{l}
U \\
P
\end{array}\right)=\left(\begin{array}{c}
F \\
f
\end{array}\right)
$$

where: $K_{T}$ and $M_{T}$ are the overall stiffness matrix and mass matrix of the structure, $K_{S}$ and $M_{S}$ are the stiffness matrix and mass matrix of the fluid domain, $L_{\mathrm{c}}$ is the coupling effect between the fluid domain and the structure, $U$ is the displacement of the node, and $F$ is the effect on the structure Excitation, $f$ is the force applied to the fluid domain.

\subsection{Material and fluid sound absorption}

The calculation unit includes the water layer and the viscoelastic layer. The damping factor of the viscoelastic material is omitted. The attenuation factor of the Young's modulus is used to represent the acoustic damping, and $\eta$ means loss factor:

$$
E^{\prime}=E \times(1+\eta i)
$$

When performing finite element modeling, the perfect matching layer (PML) can be used to simulate an infinite fluid domain and absorb the excess sound waves radiating outward from the water.

\subsection{Sound absorption coefficient}

The sound wave is incident perpendicularly from the fluid domain to the acoustic material, The reflection coefficient of sound pressure is: 


$$
R=\frac{Z_{s}-Z_{0}}{Z_{s}+Z_{0}}
$$

where $Z_{0}$ is the characteristic impedance of the fluid medium; $Z_{s}$ is the output impedance of the fluid-solid interface.

In this paper, the sound waves are incident from water, and the acoustic material is made of steel plate air backing. Because the steel plate backing and air impedance are very different, the transmitted sound energy is considered to be negligible, so the sound absorption coefficient is:

$$
\alpha=1-|R|^{2}
$$

\subsection{Finite element model verification}

Calculate the acoustic properties of the semi-infinite medium with the viscoelastic layer attached to the iron plate and water and air on both sides, as shown in Fig.1.

The density of steel plate $\rho=7700 \mathrm{~kg} / \mathrm{m}^{\wedge} 3$, Young's modulus $E=2.1 \times 10^{\wedge} 11$, Poisson' $\mathrm{s}$ ratio $\gamma=0.25$, the steel plate thickness is $1 \mathrm{~cm}$; the density of air $\rho=1.21 \mathrm{~kg} / \mathrm{m}^{\wedge} 3$, the speed of sound It is $\mathrm{c}=343 \mathrm{~m} / \mathrm{s}$. The plane wave is incident perpendicularly, the frequency of the incident wave is $0.1 \mathrm{kHz} \sim 10 \mathrm{kHz}$, and the reflection coefficient of the acoustic material is shown in Fig.2. It can be seen from Fig.2. that the analytical solution is consistent with the numerical solution, indicating that the finite element method is effective.

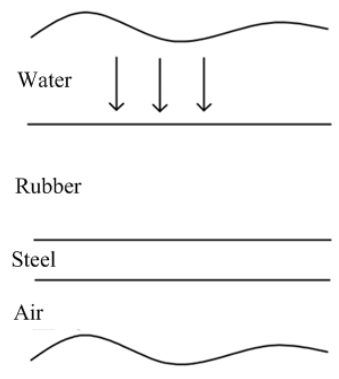

Fig. 1. Schematic diagram of backing non-cavity acoustic material.

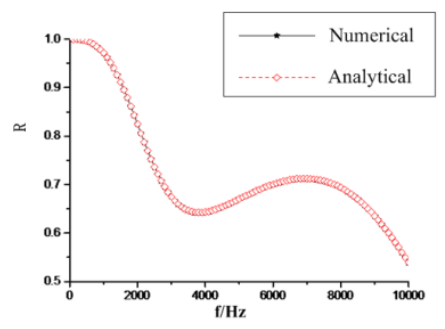

Fig. 2. Backing non-cavity acoustic material reflection coefficient.

\subsection{Introduction to optimization algorithms}

The Nelder-Mead simplex method is a direct search algorithm proposed by Nelder and Mead. The basic idea can be described as: by optimizing the simplex, the simplex gradually converges to the minimum position. In each iteration process, we calculate the corresponding function value of the objective function at each point set position of the simplex, and then sort these function values, and satisfy: 


$$
f\left(x_{1}\right) \leq f\left(x_{2}\right) \ldots \leq f\left(x_{n+1}\right)
$$

The worst point is replaced with the new best point. This iterative process is repeated until the simplex converges to the maximum value of the objective function.

\section{Numerical calculation model}

In this paper, the internal cavity structure of the acoustic material is periodically distributed. According to Bloch theory, it is known that only the finite element model of the unit cell is needed to obtain the acoustic properties of the acoustic material. The linear function of the internal cavity of the acoustic material is:

$$
h=-0.04 \times\left(\frac{r}{7}\right)^{6}+0.04
$$

The cavity radius is $\mathrm{R}=7 \mathrm{~mm}$ and the height $\mathrm{H}=40 \mathrm{~mm}$, as shown in Fig.3.:
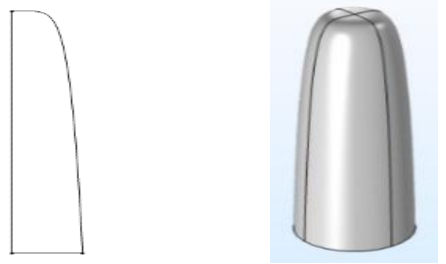

(a) Two-dimensional image (b) Three-dimensional image

Fig. 3. Cavity model.

The acoustic material model based on the finite element method is shown in Fig.4, Periodic boundary conditions need to be set at the unit cell structure and the fluid boundary to realize the simulation of the infinite acoustic material. The specific geometric dimensions and material parameters of the model are shown in Table 1 and Table 2. The inside of the acoustic material is a cavity with a height of $40 \mathrm{~mm}$, and the distance between the cavity and the upper and lower interfaces is $5 \mathrm{~mm}$. The perfect matching layer (PML) is added to the upper part of the water area to simulate the infinite water area and absorb excess sound waves. The steel plate backing is air. Since the steel plate backing and air impedance are very different, the transmitted sound energy is considered to be negligible.

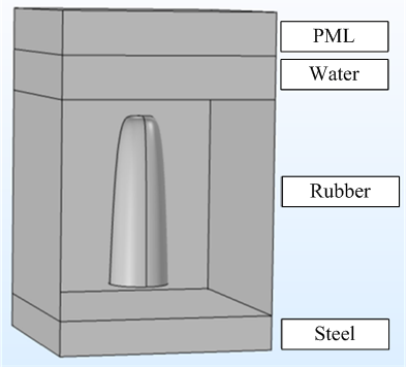

Fig. 4. Finite element model of the acoustic material.

Table 1. Material parameters.

\begin{tabular}{|c|c|c|c|c|c|}
\hline Material & $\begin{array}{c}\text { Elastic Modulus } \\
(\mathrm{Pa})\end{array}$ & $\begin{array}{c}\text { Poisson's } \\
\text { ratio }\end{array}$ & $\begin{array}{c}\text { Material loss } \\
\text { factor }\end{array}$ & density $\left(\mathrm{kg} / \mathrm{m}^{3}\right)$ & $\begin{array}{c}\text { Speed of } \\
\text { sound }(\mathrm{m} / \mathrm{s})\end{array}$ \\
\hline Steel & $1 \mathrm{e} 11$ & 0.25 & - & 7700 & - \\
\hline Rubber & $7.1 \mathrm{e} 7$ & 0.49 & 0.2 & 1100 & - \\
\hline
\end{tabular}




\begin{tabular}{|c|l|l|l|l|l|}
\hline Water & - & - & - & 1000 & 1500 \\
\hline Air & - & - & - & 1.21 & 343 \\
\hline
\end{tabular}

Table 2. Geometric parameters.

\begin{tabular}{|c|c|c|c|}
\hline Computational domain & Length $(\mathrm{mm})$ & Width $(\mathrm{mm})$ & Thickness $(\mathrm{mm})$ \\
\hline Steel & 50 & 50 & 10 \\
\hline Rubber & 50 & 50 & 50 \\
\hline Water & 50 & 50 & 10 \\
\hline PML & 50 & 50 & 10 \\
\hline
\end{tabular}

\section{Results and discussion}

\subsection{Sound absorption characteristics of the acoustic material}

The sound waves are incident from the upper waters at different angles, the incident frequency is $0.1 \mathrm{kHz} 10 \mathrm{kHz}$, the step length is $0.1 \mathrm{kHz}$, and the sound absorption coefficient of the acoustic material is shown in Fig.5 below. In this paper, the influence of external static pressure on sound absorption characteristics, the dispersion of viscoelastic material characteristics and dynamic characteristics such as temperature changes are not considered.

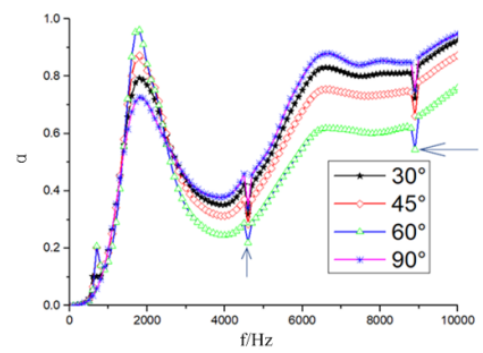

Fig. 5. The sound absorption coefficient of the acoustic material at different incident angles.

The fluctuation of the sound absorption coefficient at the arrow in Fig. 5 is caused by the formation of thin layers of rubber on the upper and lower layers of the cavity to excite bending waves. When the thickness of the thin layer increases, the cavity is reduced $(\mathrm{R}=3 \mathrm{~mm}, \mathrm{H}=20 \mathrm{~mm})$ ), the fluctuation will disappear. Fig. 6 shows the change of the sound absorption coefficient of the acoustic material with cavities of different volumes. It can be seen that when the thickness of the thin layer increases, the fluctuation of the sound absorption coefficient disappears.

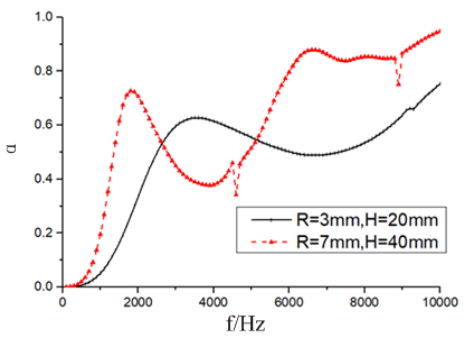

Fig. 6. Comparison of cavities with different volumes. 
It can be seen from Fig. 5 that in the range of $30^{\circ} \sim 90^{\circ}$, the peak of the first sound absorption coefficient gradually increases with the increase of the angle of incidence, and the peak of the second sound absorption decreases with the increase of the angle of incidence. In normal incidence, compared with oblique incidence, the first peak of the sound absorption coefficient is the lowest, and the second peak of the second sound absorption is the highest. At the same time, the change of the incident angle did not significantly affect the frequency position of the sound absorption peak. Taking vertical incidence as an example, the first sound absorption peak of the sound absorption coefficient of the acoustic material is around $2 \mathrm{kHz}$, and the second sound absorption peak is around $7 \mathrm{kHz}$. At each sound absorption peak frequency, the displacement of the acoustic material is shown in Fig.7:
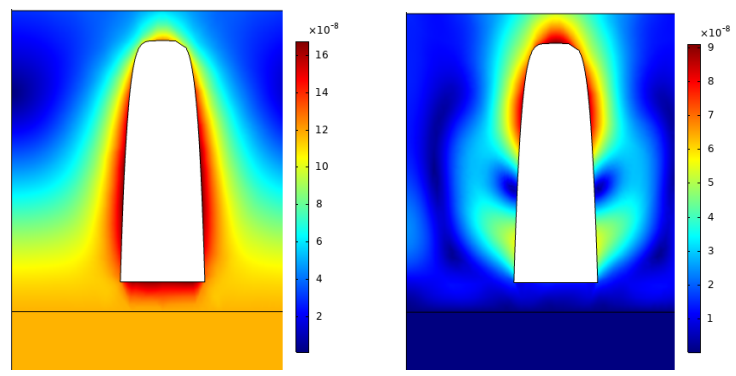

(a) Displacement diagram at the first sound absorption peak (b) Displacement diagram at the second sound absorption peak

Fig. 7. Peak displacement of sound absorption.

From the displacement diagram in Fig.7(a), it is found that, as a whole, from bottom to top, the displacement amplitude of the base part of the acoustic material gradually increases, indicating that the acoustic material is stretched or compressed as a whole. Therefore, the preliminary judgment is that due to the coupling between the backing and the acoustic material, the sound waves are reflected by the interface formed by the steel plate, the acoustic material and the air, and act on the cavity to cause the overall resonance of the sound absorption layer, thereby Reach the peak of sound absorption. Fig.7(b) shows that at this frequency point, the cavity vibration amplitude is large, while the acoustic material matrix displacement is small, indicating that the second sound absorption peak is caused by cavity resonance sound absorption.

\subsection{Optimized calculation for vertical incidence of sound waves}

This paper optimizes the design of the inner cavity line and the material of the acoustic material. By adding a deviation function to the formula (6) cavity bus, the deviation of the bus shape from the original bus shape after optimization is expressed, and the cavity bus expression after adding the deviation function for:

$$
h=-0.04 \times\left(\frac{r}{7}\right)^{6}+0.04+\Delta h
$$

where: the deviation function $\Delta h=\sum_{i=1}^{N} Q_{i}\left(\frac{r}{7}\right)^{i}\left(1-\frac{r}{7}\right)^{N-i}$

When the value of $\mathrm{N}$ in the deviation function is larger, the calculation result is more accurate, but the calculation time is longer. In this paper, $\mathrm{N}=5$ and the initial value of the deviation function coefficient $Q_{1} \sim Q_{5}=0$. 
First, compare the sound absorption coefficient before and after optimization at a single frequency point. Assuming that the incident sound wave frequency is $0.8 \mathrm{kHz}, 1 \mathrm{kHz}$ and $1.5 \mathrm{kHz}$, the optimization goal is to maximize the sound absorption coefficient at this frequency point. After optimization, the sound absorption at each frequency point The coefficient changes are shown in Table 3

Table 3. Single frequency point optimization.

\begin{tabular}{|c|c|c|}
\hline $\mathrm{f}(\mathrm{kHz})$ & $\begin{array}{c}\text { Sound absorption coefficient } \\
\text { before optimization }\end{array}$ & $\begin{array}{c}\text { Sound absorption coefficient } \\
\text { after optimization }\end{array}$ \\
\hline 0.8 & 0.06 & 0.98 \\
\hline 1 & 0.145 & 0.99 \\
\hline 1.5 & 0.532 & 0.856 \\
\hline
\end{tabular}

The shape change of the cavity bus bar after optimization is shown in Fig.8.
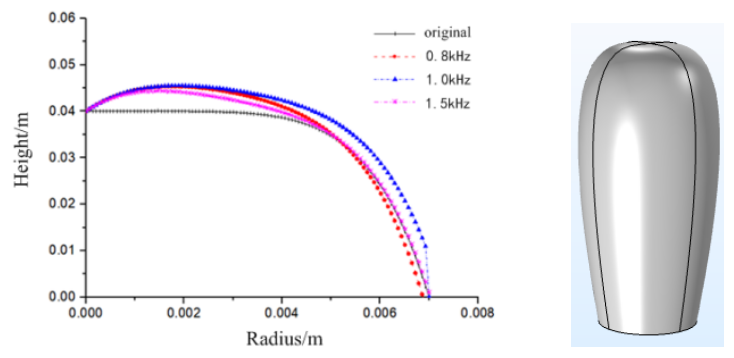

(a) Line shape comparison after optimization (b) 3D image of cavity after optimization at $1 \mathrm{kHz}$ frequency

Fig. 8. Bus line shape change after optimization.

Improving the sound absorption performance under multi-frequency points is also of great significance to the acoustic material. The following is an optimized design for this problem. The maximum sound absorption coefficient is also used as the objective function, and the cavity line shape parameters are variables. The objective function is no longer the sound absorption coefficient under a single frequency point, but the average sound absorption coefficient of the entire frequency band:

$$
\text { obj } \alpha=\sum_{n}^{N} \alpha_{n}
$$

where: $\mathrm{n}$ is the optimized initial frequency, $\mathrm{N}$ is the stop frequency, $\alpha$ is the sound absorption coefficient at different frequency points, and obj $\alpha$ is the superposition of the sound absorption coefficient at all frequency points. The optimized frequency band is $0.8 \mathrm{kHz} \sim 10 \mathrm{kHz}$, because the sound absorption coefficient fluctuates sharply at low frequencies, which has a greater impact on the sound absorption performance of the acoustic material. Set $0.5 \mathrm{kHz}$ in steps from $0.8 \mathrm{kHz}$ to $5 \mathrm{kHz}$, and $5 \mathrm{kHz} 10 \mathrm{kHz}$ in steps of $1 \mathrm{kHz}$, optimized The comparison of the front and rear sound absorption coefficients is shown in Fig.9: 


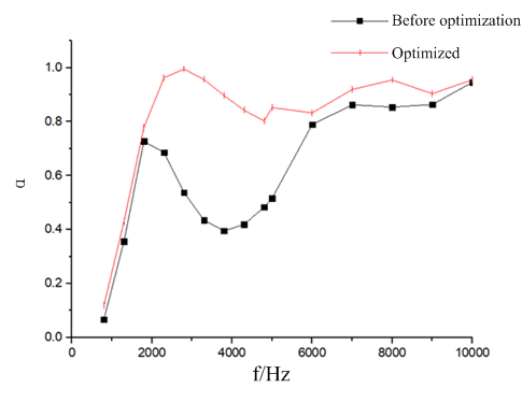

Fig. 9. Comparison of sound absorption coefficient at normal incidence.

It can be seen from Fig.9 that the sound absorption performance of the acoustic material is quite different before and after optimization, especially in the range of $2 \mathrm{kHz} \sim 5 \mathrm{kHz}$, the sound absorption performance of the acoustic material is improved the most after optimization, for example, at the frequency of $3.8 \mathrm{kHz}$, the sound absorption of the acoustic material before optimization The coefficient is 0.39 , which is 0.89 after optimization. Fig. 10 compares the displacement change of the acoustic material at $2.8 \mathrm{kHz}$ and $3.8 \mathrm{kHz}$.
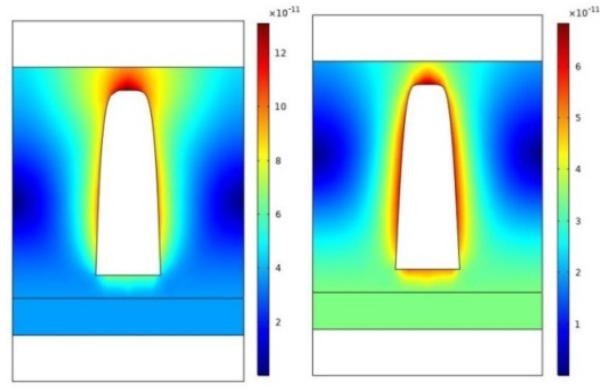

(a) Before $2.8 \mathrm{kHz}$ optimization (b) After $2.8 \mathrm{kHz}$ optimization
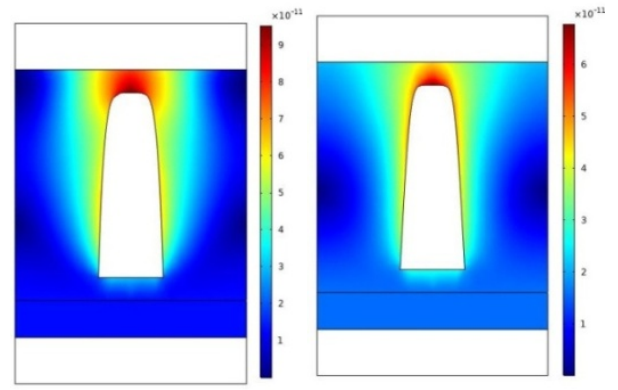

(c) Before $3.8 \mathrm{kHz}$ optimization (d) After $3.8 \mathrm{kHz}$ optimization

Fig. 10. Displacement diagram before and after optimization.

Comparing Fig.10(a)(b) the displacement map of acoustic material before and after $2.8 \mathrm{kHz}$ optimization, the displacement amplitude of the base part of the acoustic material gradually increases after optimization. This is due to the coupling between the backing and the acoustic material, which makes the sound waves be affected by the steel plate and the acoustic material. The interface formed by the material and air reflects back and acts on the cavity, causing the acoustic material to be stretched or compressed as a whole, thereby improving the optimized sound absorption performance. 
Compare Fig.10(c)(d) the displacement map of $3.8 \mathrm{kHz}$ acoustic material before and after optimization. At this frequency, the displacement of the top of the cavity inside the acoustic material decreases after optimization, but the displacement amplitude of the base part increases, indicating that at this frequency point Next, the improvement of the sound absorption performance after optimization depends on the vibration of the acoustic material.

\section{Conclusion}

In this paper, an acoustic material with a steel plate backing and a cavity is used as a research pair phenomenon. A finite element model is established by the finite element mothed to solve the sound absorption characteristics of the acoustic material, and the sound absorption coefficient of the acoustic material under different incident angles is compared and analyzed. Then use the acoustic material and the internal cavity of the acoustic material as design variables, and take the maximum sound absorption coefficient at the corresponding frequency point as the objective function, consider different frequencies and incident angles, establish optimized design models, and calculate the corresponding optimal geometric shapes And material parameters, the following conclusions can be drawn:

(1) Due to the existence of the cavity inside the acoustic material and the coupling between the backing and the acoustic material, the sound absorption coefficient of the sound-absorbing acoustic material has two peaks. The first peak increases with the increase of the incident angle, and the second peak decreases with the increase of the incident angle, and the change of the incident angle does not significantly affect the frequency position of the sound absorption peak;

(2) In this paper, by adding a deviation function to the cavity bus, the cavity line shape can be optimized at different incident angles and different frequencies, and the limitations of the previous need to modify the cavity line shape by experience can be changed;

(3) The plane wave frequency is $0.8 \mathrm{kHz} \sim 10 \mathrm{kHz}$ in the given frequency band, and it is incident perpendicularly from the top of the acoustic material. After optimization, the sound absorption performance is improved at $2 \mathrm{kHz} \sim 6 \mathrm{kHz}$, and the peak and valley values of the sound absorption coefficient are optimized;

(4) From the comparison chart of the displacement of the acoustic material before and after optimization, it can be seen that the optimization changes the internal cavity line shape of the acoustic material and the material parameters of the acoustic material, resulting in changes in the resonance frequency of the cavity and the acoustic material, thereby improving the sound absorption performance of the acoustic material.

Through optimizing the design, this paper improves the sound absorption coefficient of the cavity-containing acoustic material at a given frequency band and a given incident angle, and provides a certain reference value for the design of cavity-containing acoustic material.

\section{References}

1. Zhang Hongjun, Qiu Bohua, Shi Lei, He Peng. Current status and development trend of anechoic tile technology[J]. Ship Science and Technology, 2001(04): 6-10+14.

2. Luo Zhong, Zhu Xi, Lin Zhituo, Wang Weizhong. Research progress on the structure and sound absorption mechanism of underwater sound-absorbing coatings[J]. Ship Science and Technology, 2009, 31(08): 23-30.

3. Wen J,Zhao H,Lv L,et al.Effects of locally resonant modes on underwater sound absorption in viscoelastic materials[J].The Journal of the Acoustical Society of America.2011,130(3):1201-1208 
4. Hennion A C,Bossut R,Decarpigny $\mathrm{J}$ N,el al.Analysis of the scattering of a plane acoustic wave by aperiodic elastic structure using the finite element method: Application to compliant tube gratings[J].The Journal of the Acoustical Society of America.1990,87(5):1861-1870.

5. Sharm G S,Skvortsov A,MacGillivray I.Acoustic performance of periodic steel cylinders embedded in a viscoelastic medium[J].Iournal of sound and Vibration.2019,443:652-665.

6. Sharm G S,Skvortsov A,MacGillivray I, et al.Sound absorption by rubber coatings with periodic voids and hard inclusions[J].Applied Acoustic.2019,143:200-210

7. Sharm G S,Skvortsov A,MacGillivray I,Sound transmission through a periodically volided soft elastic medium submergrd in water[J].Wave Motion.2017,443:101-112

8. Changzheng Ye, Xuewei Liu, Fengxian Xin and TianJianLu. Influence of hole shape on sound absorption of underwater anechoic layers[J]. Journal of Sound and Vibration 426 (2018) 54-74.

9. Ivansson S M.Numerical design of alberich anechoic coatings with superellipsoidal cavities of mixed sizes[J].The Journal of the Acoustical Society of America.2008,124(4):1974-1984.

10. Hennion A C,Bossut R,Decarpigny $\mathrm{J}$ N,el al.Analysis of the scattering of a plane acoustic wave by aperiodic elastic structure using the finite element method: Application to compliant tube gratings[J].The Journal of the Acoustical Society of America.1990,87(5):1861-1870.

11. Tan Hongbo, Zhao Hong, $\mathrm{Xu}$ Haiting. Analysis of acoustic characteristics of viscoelastic layer with periodic cavity distribution by finite element method[J]. Acta Acoustics, 2003(03):277-282.

12. Ivansson $\mathrm{S}$ M.Anechoic coatings obtain from two-and three-dimensional monopole resonance diffraction gratings[J]. The Journal of the Acoustical Society of America.2012,131(4):2622-2637.

13. Shang Chao, Zhang Jiazhong, Cao Wei, Yu Kaiping. Research on sound absorption performance of hybrid cavity structure cover layer and its optimal design[J]. Vibration and Shock, 2011, 30(08): 217-220. 